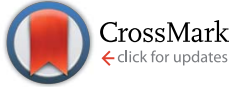

Cite this: RSC Adv., 2017, 7, 12089

Received 18th January 2017 Accepted 12th February 2017

DOI: 10.1039/c7ra00767a

rsc.li/rsc-advances

\title{
Hierarchical S-doped porous carbon derived from by-product lignin for high-performance supercapacitors $\dagger$
}

\begin{abstract}
Jingyang Tian, Zhangming Liu, Zhenghui Li, Wenguang Wang and Haiyan Zhang*
For the first time, hierarchical S-doped porous carbon was fabricated using sodium lignosulfonate as a carbon and sulfur precursor through a simple template technique. The obtained carbon has a high surface area $\left(1054 \mathrm{~m}^{2} \mathrm{~g}^{-1}\right)$, high pore volume $\left(1.73 \mathrm{~cm}^{3} \mathrm{~g}^{-1}\right)$, high sulfur content (2.9 wt\%), and interconnected open macropores that can provide many storage sites, pseudocapacitance, and short transport paths for ions. The as-obtained S-PC-L-900 exhibits a high specific capacitance $\left(328 \mathrm{~F} \mathrm{~g}^{-1}\right)$ at $0.2 \mathrm{~A} \mathrm{~g}^{-1}$, excellent rate performance with $73 \%$ capacitance retention after a current density increasing from 0.5 to $20 \mathrm{~A} \mathrm{~g}^{-1}$ and cycling stability, with 97\% capacitance retention after 10000 cycles. Additionally, a symmetric supercapacitor based on S-PC-L-900 delivers a high energy density $(6.9 \mathrm{~W} \mathrm{~h}$ $\mathrm{kg}^{-1}$ ) at $50 \mathrm{~W} \mathrm{~kg}^{-1}$.
\end{abstract}

\section{Introduction}

With exacerbated energy and environmental problems such as pollution, fossil fuel depletion, and global warming, sustainable and renewable energy materials have become more widespread. ${ }^{1}$ Supercapacitors, also known as electrochemical capacitors, have attracted most attention because of their high power density, fast charge and discharge, and long cycle life. ${ }^{2-5}$ Electrochemical capacitors can be categorized into electrical double-layer capacitors (EDLCs) and pseudocapacitors according to the energy storage mechanism. ${ }^{6,7}$ The energy storage of EDLCs is based on the electrostatic storage of electrical energy achieved by separation of charge in a Helmholtz double layer at the electrode/electrolyte interface. Therefore, the increase in the electrode surface area, which is accessible to electrolyte ions, can lead to higher capacitance. Another important factor is the surface properties and porosity of the electrode, such as pore size. ${ }^{8}$ On the other hand, the electrochemical storage of electrical energy for pseudocapacitors is dominated by faradaic charge transfer that occurs between the electrodes and electrolyte, which leads to reversible redox reactions.

Recently, porous carbon materials, one of the carbonaceous materials, have been widely used as electrodes for EDLCs mainly because of their high surface area, good conductivity, well-developed pore structure, and high physicochemical stability., ${ }^{\mathbf{9} 10}$ Most of the porous carbon consists of narrow

College of Materials and Energy, Guangdong University of Technology, Guangzhou 51006, China. E-mail: hyzhang@gdut.edu.cn; Fax: +86 20 39322570; Tel: +86 20 39323232

$\dagger$ Electronic supplementary information (ESI) available. See DOI: 10.1039/c7ra00767a micropores $(<1 \mathrm{~nm})$ which can exhibit a large BET surface area. However, these granular powders of porous carbon can result in large diffusion paths of ions. This is a serious drawback, as it limits the transport of ions in the pores, which leads to a sharp reduction of EDLCs performance at high current densities. ${ }^{\mathbf{1 1}}$ Reducing the effective diffusion paths such as hierarchical structure containing macropores, mesopores, and micropores is one useful strategy. The macropores and mesopores can respectively act as ion-buffering reservoirs and channels for rapid transport of ions. Meanwhile, micropores provide abundant locations for charge accommodation. Therefore, preparation of hierarchical porous carbon containing a certain amount of macropores and mesopores is important to EDLC systems.

The preparation of porous carbon usually involves complex processes during synthesis, while most of them require a series of harsh chemical treatments. However, direct pyrolysis of abundant and renewable carbon precursors such as biowaste $^{12,13}$ and biomass, ${ }^{7,10}$ has been proven to be a sustainable low-cost strategy. ${ }^{\mathbf{1 4}}$ The good performance of supercapacitor electrodes produced through carbonization of biomass such as waste coffee grounds, ${ }^{8}$ seaweeds, ${ }^{15}$ and rice husks ${ }^{16}$ has been demonstrated. Lignin is one of the most abundant components of biomass ranks, second to cellulose. After the pulping process, lignin is obtained as a non-valuable by-products; its combustion to obtain energy ${ }^{17}$ results in serious air pollution. Therefore, many studies have explored transformation of lignin into highvalue-added products. ${ }^{18,19}$

Doping of heteroatoms into carbon materials is an efficient way of improving the intrinsic chemical and electrical properties because of the introduced extrinsic defects that function as electron acceptors. ${ }^{20-22}$ In a recent study, carbon materials have been doped with heteroatoms such as nitrogen, phosphorus, 
sulfur, and boron via single or dual methods. ${ }^{23-26}$ In particular, sulfur-doped materials with wider band gap have drawn great attention because of the electron-withdrawing property of $\mathrm{S}^{21}$ Much researches have focused on the application of S-doped carbon materials, such as cathode catalysts, ${ }^{27} \mathrm{Li}$ ion batteries, ${ }^{28,29}$ electrochemical capacitors, ${ }^{30,31}$ and $\mathrm{H}_{2}$ storage. ${ }^{32}$

Sulfur-doped carbon is generally synthesized through a complex procedure that includes mixing of the sulfurcontaining and carbon precursors. Most of the sulfurcontaining precursors such as benzyl disulfide, ${ }^{33}$ hydrogen sulfide $^{34}$ and carbon disulfide ${ }^{21}$ are toxic. One novel environment-friendly way of obtaining sulfur-doped materials is utilizing precursors that contain both carbon and sulfur, which can also reduce the number of mixing steps. ${ }^{35}$

In this paper, we present a simple environment-friendly procedure for the synthesis of hierarchically S-doped porous carbon (S-PC-L) via direct carbonization of sulfur- and carboncontaining sodium lignosulfonate with silica templates. After removal of inorganic impurities, L-S-PC was obtained. Compared with undoped porous carbon prepared by carbonization of sucrose (PC-S), ${ }^{36}$ L-S-PC shows higher specific capacitance due to the particular hierarchical structure, which contains micropores as well as many macropores and mesopores. This approach of synthesis of sulfur-doped porous carbon not only provides an economical material for EDLCs, but also supplies a green alternative technique to those using toxic sulfur precursors.

\section{Experimental section}

\subsection{Synthesis of the hierarchically S-doped porous carbon (S- PC-L)}

Similar to a previous report, ${ }^{37} \mathrm{~L}-\mathrm{S}$-PC was prepared through the following method. Sodium lignosulfonate (LS, $2.0 \mathrm{~g}$ ) and sulfuric acid ( $3 \mathrm{ml}, 1 \mathrm{M}$ ) were transferred to a vial and then stirred for about $0.5 \mathrm{~h}$ until the LS completely dissolved. Tetraethyl orthosilicate (TEOS, $4 \mathrm{ml}$ ) was then added quickly under vigorous stirring for $3 \mathrm{~h}$ until the mixture became homogenous. The vial was placed in an oven and dried at $40{ }^{\circ} \mathrm{C}$ for $48 \mathrm{~h}$. Subsequently, the product was pre carbonized at $100{ }^{\circ} \mathrm{C}$ and $160{ }^{\circ} \mathrm{C}$ for $6 \mathrm{~h}$, respectively. Afterward, the obtained gel monolith was placed in a tube furnace and carbonized at $700-900{ }^{\circ} \mathrm{C}$ for $3 \mathrm{~h}$ at a heating rate of $5{ }^{\circ} \mathrm{C} \min ^{-1}$ under $\mathrm{N}_{2}$ protection. Eventually, the carbon solid was etched with excess $\mathrm{HF}(40 \%)$ to remove the silica template and washed several times until the $\mathrm{pH} 7$ was reached. The material was then dried at $105{ }^{\circ} \mathrm{C}$ overnight in an oven. The harvested samples were labeled as S-PC-L700, S-PC-L-800, and S-PC-L-900 for different carbonization temperature. Porous carbon material derived from sucrose through the same method at $900{ }^{\circ} \mathrm{C}$ (PC-S-900) was used as control sample. The entire preparation process of S-doped porous carbon material is shown in Scheme 1.

\subsection{Material characterization}

Images of the morphology of the prepared porous carbon were obtained using a field-emission scanning electron microscope
(FE-SEM; Ultra55, Carl Zeiss, Germany) and field-emission transmission electron microscope (FE-TEM; JEM-2100F, Japan Electronics Corporation, Japan). Raman spectra were obtained on a Raman spectrometer (LabRAM Aramis, HORIBA Jobin Yvon, France) at $532 \mathrm{~nm}$ wavelength. X-ray photoelectron spectroscopy (XPS; Amicus, Shimadzu, Japan) and element analysis (EA; Vario EL Cube, Elementar, Germany) were used to examine the chemical composition. X-ray diffraction (XRD; Rigaku Corporation, Japan) patterns obtained using $\mathrm{Cu} \mathrm{K} \alpha$ radiation were used to investigate the ordering of porous carbon. The Brunauer-Emmett-Teller (BET; Micromeritics 2460) surface area and pore size distribution were calculated from nitrogen sorption measurements at $-196{ }^{\circ} \mathrm{C}$.

\subsection{Preparation of electrodes}

The working electrodes were prepared by mixing the asprepared porous carbon S-PC-L-700, S-PC-L-800, S-PC-L-900, and PC-S-900 with acetylene black and polytetrafluoroethylene solution $(60 \mathrm{wt} \%)$ in ethanol at a mass ratio of $8: 1: 1$. The slurry was then pressed onto nickel foam at $10 \mathrm{MPa}$ and dried at $80{ }^{\circ} \mathrm{C}$ overnight in an oven. The symmetric two-electrode cells were formed by placing two electrodes with exactly the same mass in a coin cell. Glassy fibrous films and $6 \mathrm{M} \mathrm{KOH}$ aqueous solution were used as separator and electrolyte respectively. The electrodes were soaked in electrolyte for $10 \mathrm{~h}$ before assembly. Finally, the cell was pressed at $10 \mathrm{MPa}$ pressure for $10 \mathrm{~s}$ to complete the assembly.

\subsection{Electrochemical characterization}

Electrochemical measurements of the carbon electrode in different aqueous solution were carried out on a CHI $660 \mathrm{E}$ (Shanghai CHI Instruments Corporation, China) electrochemical workstation using a standard three-electrode system. The prepared electrode was used as working electrode while platinum foil was used as counter electrode. $\mathrm{Hg} / \mathrm{HgO}$ electrode was used as reference electrode in $\mathrm{KOH}$ aqueous solution while $\mathrm{Hg} / \mathrm{Hg}_{2} \mathrm{SO}_{4}$ was used in $\mathrm{H}_{2} \mathrm{SO}_{4}$ and $\mathrm{Na}_{2} \mathrm{SO}_{4}$ aqueous solution, respectively. Cyclic voltammetry (CV) was performed at scan rates of $5-2000 \mathrm{mV} \mathrm{s}^{-1}$. Galvanostatic charge/discharge (GCD) tests were done at different densities. Electrochemical impedance spectroscopy (EIS) measurements were performed in a frequency range of $0.05 \mathrm{~Hz}$ to $100 \mathrm{kHz}$ at open-circuit potential, using an AC perturbation of $5 \mathrm{mV}$. Electrochemical measurements on the symmetric two-electrode cells were done using a Neware battery testing system (CT-3008, NEWARE Electronics Corporation, China).

The specific capacitance of the electrodes was determined from galvanostatic measurement based on the following equation: ${ }^{7}$

$$
C=\frac{I_{\mathrm{d}} \times \Delta t}{\Delta V \times m}
$$

where $C$ is specific capacitance, $I_{\mathrm{d}}$ is the constant current, $\Delta t$ is the discharge time, and $\Delta V$ is the discharge voltage range (excluding the $I R$ drop). $m$ is the mass of a single active electrode 


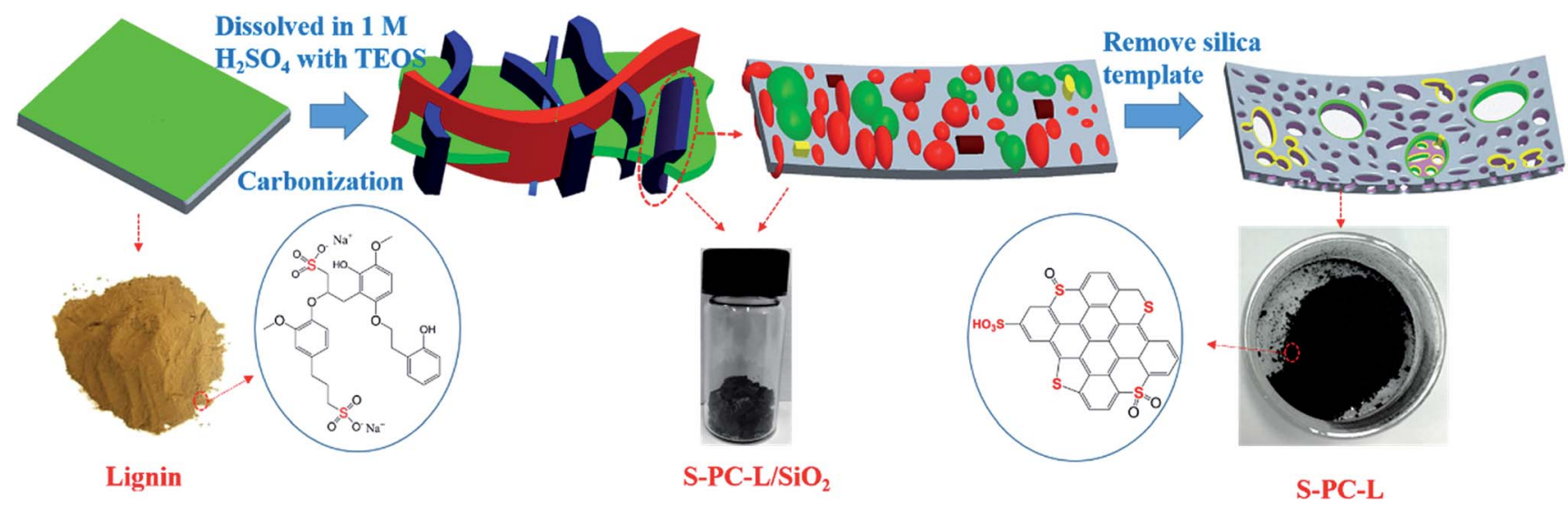

Scheme 1 Schematic of the preparation process of S-doped porous carbon material.

in the three-electrode system; in the two-electrode cell, it is the mass of both electrodes.

The energy density $E$ and power density $P$ were calculated according to the following equations:

$$
\begin{gathered}
E=\frac{1}{2} C V^{2} \\
P=\frac{E}{\Delta t}
\end{gathered}
$$

where $C$ is the specific capacitance of the supercapacitor measured from eqn (1), $V$ is the discharge voltage range (excluding the $I R$ drop), and $\Delta t$ is the discharge time.

\section{Results and discussion}

\subsection{Characterization of porous carbon}

The transformation of sodium lignosulfonate into hierarchically S-doped porous carbon mainly containing carbonization and acid washing is depicted in Scheme 1. To investigate the effect of carbonization temperature on sulfur content and combination type, we obtained S-PC-L-700, S-PC-L-800, and S-PC-L-900 by carbonization of sodium lignosulfonate at 700,800 , and $900{ }^{\circ} \mathrm{C}$ respectively. The control sample, PC-S-900, was also obtained by carbonization of sucrose at $900{ }^{\circ} \mathrm{C}$. Fig. 1 shows the morphological characterization results and microstructure of S-PC-L900. The sample possesses a typical free-standing 2D architecture consisting of many overlapped and scrolled nanosheets, as shown in Fig. 1c. The FE-SEM images (Fig. 1a and b) illustrate the high porosity and unique hierarchical porous network with a sponge-like structure inside the sample, indicating packing of the nanosheets. Besides the numerous mesopores and micropores, there are random macropores ranging from $50-300 \mathrm{~nm}$ inside S-PC-L-900 (Fig. 1d and e). Fig. 1e is the high-resolution TEM (HRTEM) image of the area marked 1 in Fig. 1d, which shows feeble lattice fringes of the nanosheet resulting from structural defects in the carbon crystal lattice due to the large atomic size of S. ${ }^{38}$ Fig. 1e suggests that the nanosheet consists of a few coupled layers with a $d$ spacing of $0.38 \mathrm{~nm}$, which is slightly larger than that of graphite $(0.33 \mathrm{~nm})$. This may due to the incorporation of the large heteroatoms ( $\mathrm{O}$ and $\mathrm{S}$ ) and carbon matrixes that protrude out of the graphene plane and increase the bond length. ${ }^{39}$ The selected-area electron diffraction pattern of S-PC-L-900 (inset of Fig. 1e) shows a typical dispersive ring-like pattern indicating the polycrystalline nature of the sample. The pattern arises from the merging of the diffraction spots because of the large number of layers in the selected area. ${ }^{40,41}$ EDS elemental mapping (Fig. 1f) shows that $\mathrm{C}, \mathrm{O}$ and $\mathrm{S}$ are homogeneously distributed throughout the entire area of the samples. Similar morphologies of S-PC-L-800 may also be observed (Fig. S1c and fï); for PC-S-900 and S-PC-L-700, no sponge-like structure and fewer macropores can be observed in the HRSEM images (Fig. S1a and $\mathrm{b} \dagger$ ) and HRTEM images (Fig. S1d and $\mathrm{e}^{\dagger}$ ) respectively. The surfaces are smooth and some fragments assemble in S-PC-L-700.

The XRD spectrum of all samples (Fig. S2 $\dagger$ ) reveal two broad peaks at $2 \theta$ angles of $23^{\circ}$ and $43^{\circ}$, which are due to the amorphous carbon structure (002) and ordered hexagonal carbon structure (100), respectively. This result reveals the formation of the amorphous structure of porous carbon even at high carbonization temperature. This feature is consistent with the porous structure shown in Fig. 1.

Nitrogen adsorption-desorption analysis was used to determine the surface area and pore size distribution. As shown in Fig. 2a, all samples exhibit typical type IV isotherms with hysteresis loops. However, the remaining samples show similar hysteresis loops in the medium-pressure regions, while S-PC-L900 show both medium- and high-pressure regions. The curve at a relative pressure of $0.45-0.75$ can be classified as a typical model for mesoporous materials while that above 0.75 can be attributed to the multilayer adsorption in the macropores ${ }^{42}$ It improves the abundance of macropores formed in S-PC-L-900, consistent with the SEM images. Fig. $2 \mathrm{~b}$ shows the pore size distributions of samples. PC-S-900 has mesopore distributions similar to those of S-PC-L-700. The mesopore size distributions of the S-PC-L samples become broader and obviously shift to larger pore diameters with increasing carbonization temperature. The micropore volume of all the samples are similar.

The physicochemical properties of all samples are compiled in Table 1. PC-S-900 has the highest specific surface area (1330 

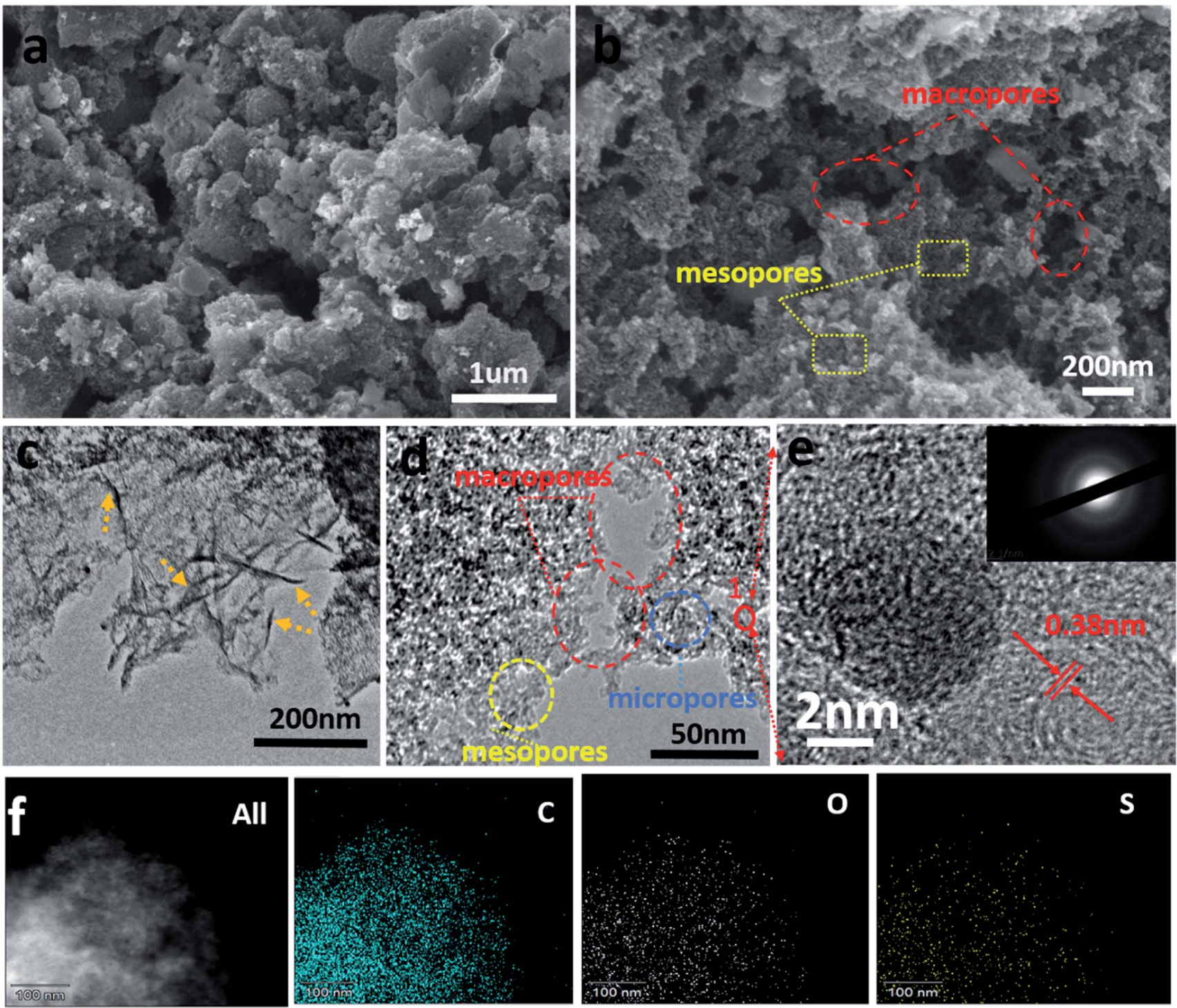

Fig. 1 Morphological characterization results for S-PC-L-900. (a and b) FE-SEM, (c and d) FE-TEM, (e) high-resolution FE-SEM images, (f) EDS elemental maps. Red, yellow, and blue rings in (b) and (d) indicate the macropores, mesopores, and micropores of S-PC-L-900 respectively; the orange arrows in (c) show the graphene-like sheet wrinkle of S-PC-L-900; the inset in (e) is the SAED pattern of the area labeled 1 in panel (d).
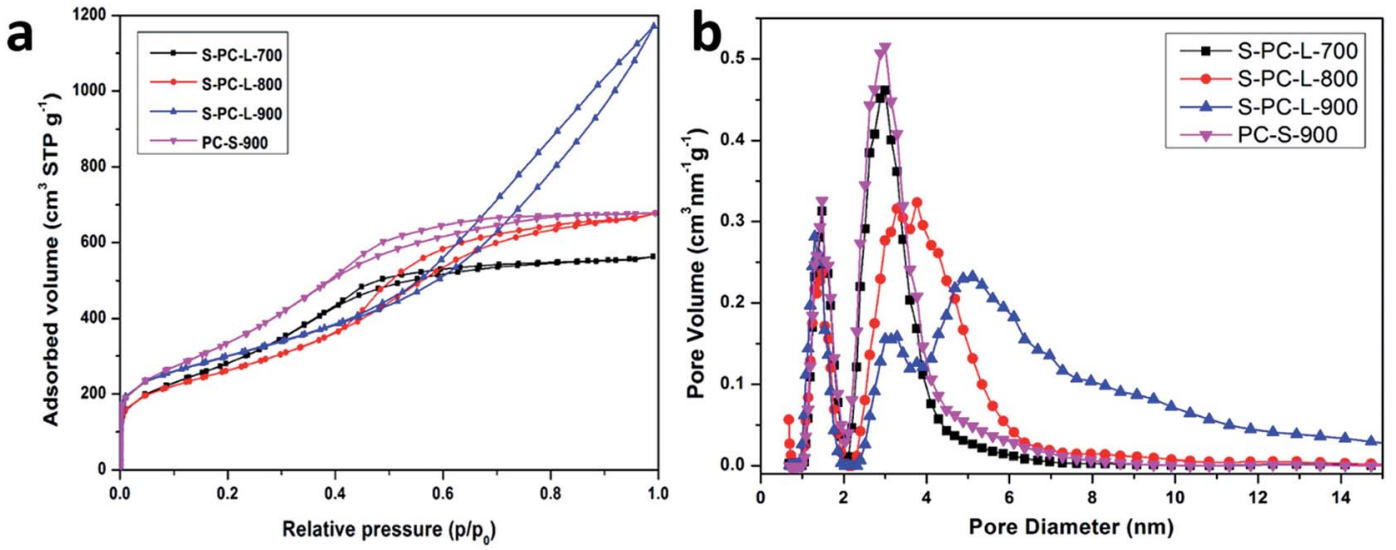

Fig. 2 (a) Nitrogen adsorption-desorption isotherms; (b) pore size distributions of the samples calculated by non local density functional theory (NLDFT). 
Table 1 Physicochemical properties of the samples

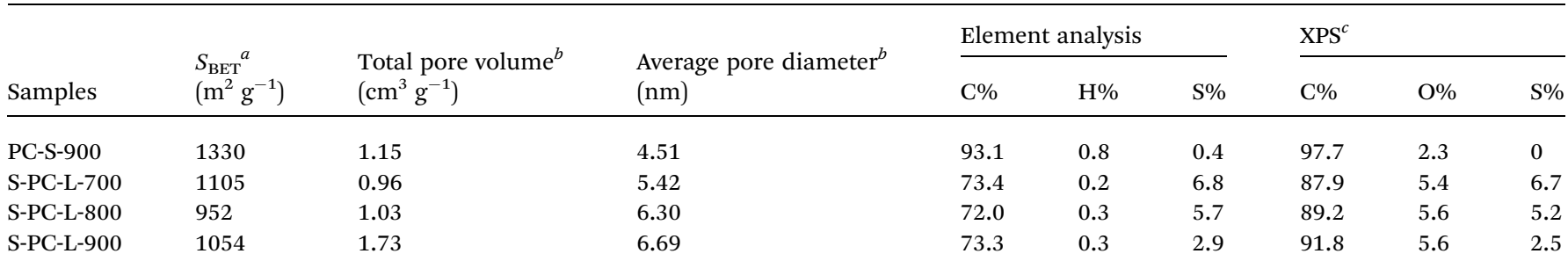

${ }^{a}$ Brunauer-Emmett-Teller (BET) surface area. ${ }^{b}$ Density functional theory (DFT). ${ }^{c}$ Weight percentage of C, O, and S obtained from XPS.

$\left.\mathrm{m}^{2} \mathrm{~g}^{-1}\right)$. All S-PC-L samples have similar specific surface areas $\left(1105,952\right.$, and $1054 \mathrm{~m}^{2} \mathrm{~g}^{-1}$ for the S-PC-L-700, -800, and -900, respectively). However, the S-PC-L-900 had the highest total pore volume $\left(1.73 \mathrm{~cm}^{3} \mathrm{~g}^{-1}\right)$ and average pore diameter $(7.33 \mathrm{~nm})$. It is possible that the carbon network is not fully developed at low carbonization temperature, resulting in low pore volume and porosity (Fig. S1 $\dagger$ ). With the increase in carbonization temperature, carbon particles can form an interconnected sponge-like structure, as shown in Fig. 1b. ${ }^{43}$

Further structural information on the samples was obtained by Raman spectra analysis. Two typical peaks at 1335 and 1590 $\mathrm{cm}^{-1}$ for all samples can be observed; these correspond to $\mathrm{D}$ and $\mathrm{G}$ bands, respectively (Fig. 3). The $\mathrm{G}$ band is associated with ideal graphitic carbon structure, whereas the $\mathrm{D}$ band corresponds to disordered carbon. ${ }^{43}$ The ratios of the $\mathrm{G}$ band to the D band $\left(I_{\mathrm{G}} / I_{\mathrm{D}}\right)$ for S-PC-L-700, -800 , and -900 are $0.98,1.04$, and 1.11 , respectively. This is due to the higher carbonization temperature, which contributes to the formation of graphitic $\mathrm{sp}^{2}$ carbons. ${ }^{8}$

In order to explore the structural properties of the samples, four different peaks in the Raman spectra were obtained at $1163,1332,1499$, and $1590 \mathrm{~cm}^{-1}$, which correspond to $\mathrm{D}_{4}, \mathrm{D}_{1}$, $\mathrm{D}_{3}$, and $\mathrm{G}$ bands, respectively (Fig. $\mathrm{S} 3 \mathrm{a}-\mathrm{c} \dagger$ ). $\mathrm{D}_{1}$ and $\mathrm{D}_{4}$ bands indicate disordered graphitic carbon while the $\mathrm{D}_{3}$ band indicates amorphous carbon. ${ }^{\mathbf{4 4 , 4 5}}$ As shown in Fig. S3d, $\dagger$ the percentage of amorphous carbon decreases while percentage of ideal graphitic carbon increases with increasing carbonization

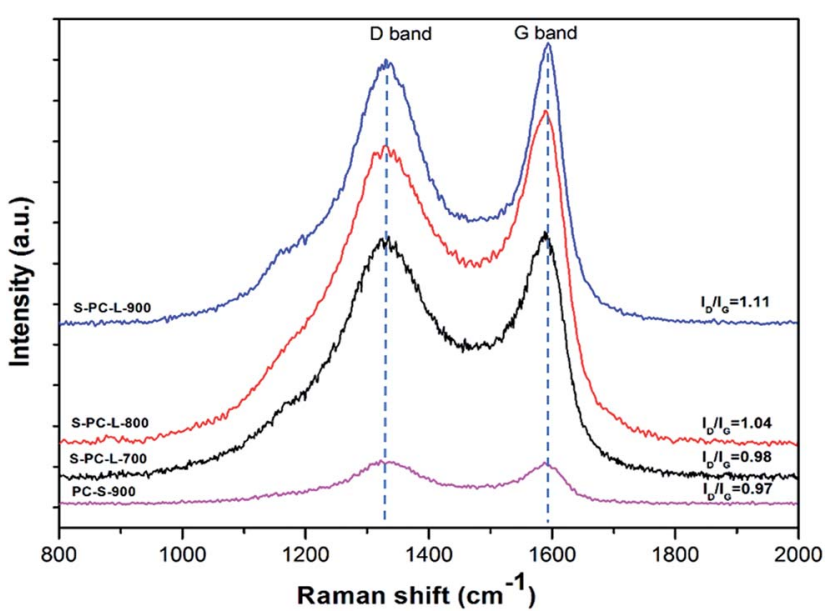

Fig. 3 Raman spectra of the samples. temperature. The results demonstrate that higher carbonization temperature can induce graphitization. ${ }^{46}$

X-ray photoelectron spectroscopy (XPS) was utilized to obtain chemical composition of the elements. The high-resolution $\mathrm{C} 1 \mathrm{~s}$ spectrum of S-PC-L-900 (Fig. 4a), -700 (Fig. S4a†), and -800 (Fig. S4c $\dagger$ ) can be fitted with peaks at $284.8 \mathrm{eV}$ (corresponding to $\mathrm{C}-\mathrm{C}$ and $\mathrm{C}-\mathrm{S}), 286.5 \mathrm{eV}(\mathrm{C}-\mathrm{O}$ and $\mathrm{C}-\mathrm{S}), 287.2 \mathrm{eV}(\mathrm{C}=\mathrm{O}), 289.1 \mathrm{eV}$ $\left(-\mathrm{CO}^{2-}\right)$, and $291.2 \mathrm{eV}\left(\pi-\pi^{*}\right) .{ }^{47,48}$ The appearance of the $\pi-\pi^{*}$ shakeup satellite shows carbonization and aromatization features, which suggest good electrical conductivity of the materials. ${ }^{49}$ Fig. $4 \mathrm{~b}$ is the corresponding high-resolution $\mathrm{S} 2 \mathrm{p}$ spectrum of S-PC-L-900, which can be deconvoluted into two types of doubles centered at $164 \mathrm{eV}\left(\mathrm{S} 2 \mathrm{p}_{3 / 2}\right)$ and $165.3 \mathrm{eV}$ (S $2 \mathrm{p}_{1 / 2}$ ) characteristic of thiophene sulfur (such as $\mathrm{C}-\mathrm{S}-\mathrm{C}$ ). ${ }^{\mathbf{5 0 , 5 1}}$ The peak at $168.8 \mathrm{eV}$ corresponding to sulfone bridges $\left(\mathrm{C}-\mathrm{SO}_{x}-\mathrm{C}\right)$ is also observable. The percentage of thiophene $\mathrm{S}$ increases while the sulfone $\mathrm{S}$ decreases with increasing carbonization temperature (Fig. $4 \mathrm{~b}, \mathrm{~S} 4 \mathrm{~b}$ and $\mathrm{d} \dagger$ ). Element analysis reveals that S-PC-L carbonized at $700{ }^{\circ} \mathrm{C}$ contains $6.8 \mathrm{wt} \%$ sulfur (Table 1). With the increase in carbonization temperature to $800{ }^{\circ} \mathrm{C}$ and $900{ }^{\circ} \mathrm{C}$, the $\mathrm{S}$ content decreases to 5.7 and $2.9 \mathrm{wt} \%$, respectively. This may due to the decrease in sulfate, which is unstable at high temperature. ${ }^{50}$ Comparison by elemental analysis using XPS reveals that the bulk $S$ content of the samples is higher than the surface content. This is understandable in that the surface functionalities have greater chance of elimination during carbonization. ${ }^{46}$

\subsection{Electrochemical performance}

The electrochemical performance of the samples was analyzed using $6 \mathrm{M} \mathrm{KOH}$ aqueous electrolyte in a three-electrode system. Fig. 5a shows the $\mathrm{CV}$ measurements at a scan rate of $50 \mathrm{mV} \mathrm{s}^{-1}$ in the potential range of -1 to $0 \mathrm{~V}$. The $\mathrm{CV}$ curves for S-PC-L-700 exhibits a CV curve that is markedly more distorted than those of other samples. It may due to the low pore volume and abundant amorphous carbon. The samples of S-PC-L-800, -900, and PC-S-900 exhibit a nearly rectangular shape, which is characteristic of ideal supercapacitors. ${ }^{52}$ S-PC-L-900 shows the highest specific capacitance and obvious redox peaks as compared with the other samples. This may have resulted from the higher percentage of graphitic carbons and thiophene sulfur of S-PC-L-900. The decrease in inactive species (sulfate) may lead to exposure of more reversible pseudo-sites, which can further improve the capacitor performance. ${ }^{42}$ Firstly, higher 

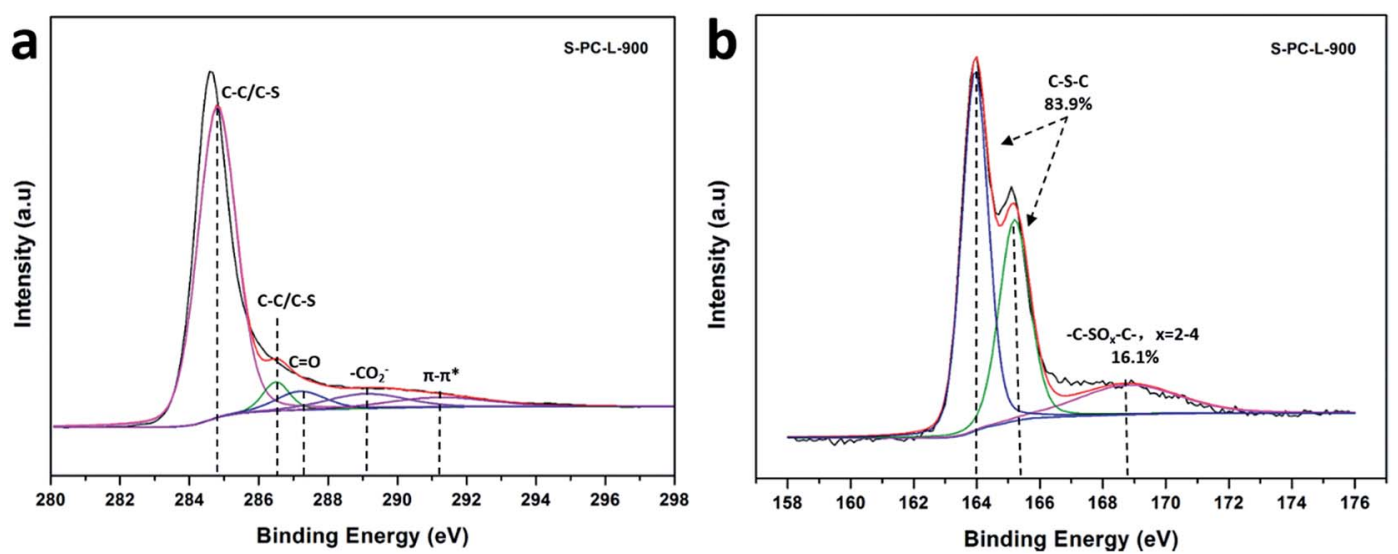

Fig. 4 High-resolution C 1s (a) and S 2p (b) XPS spectra of S-PC-L-900.
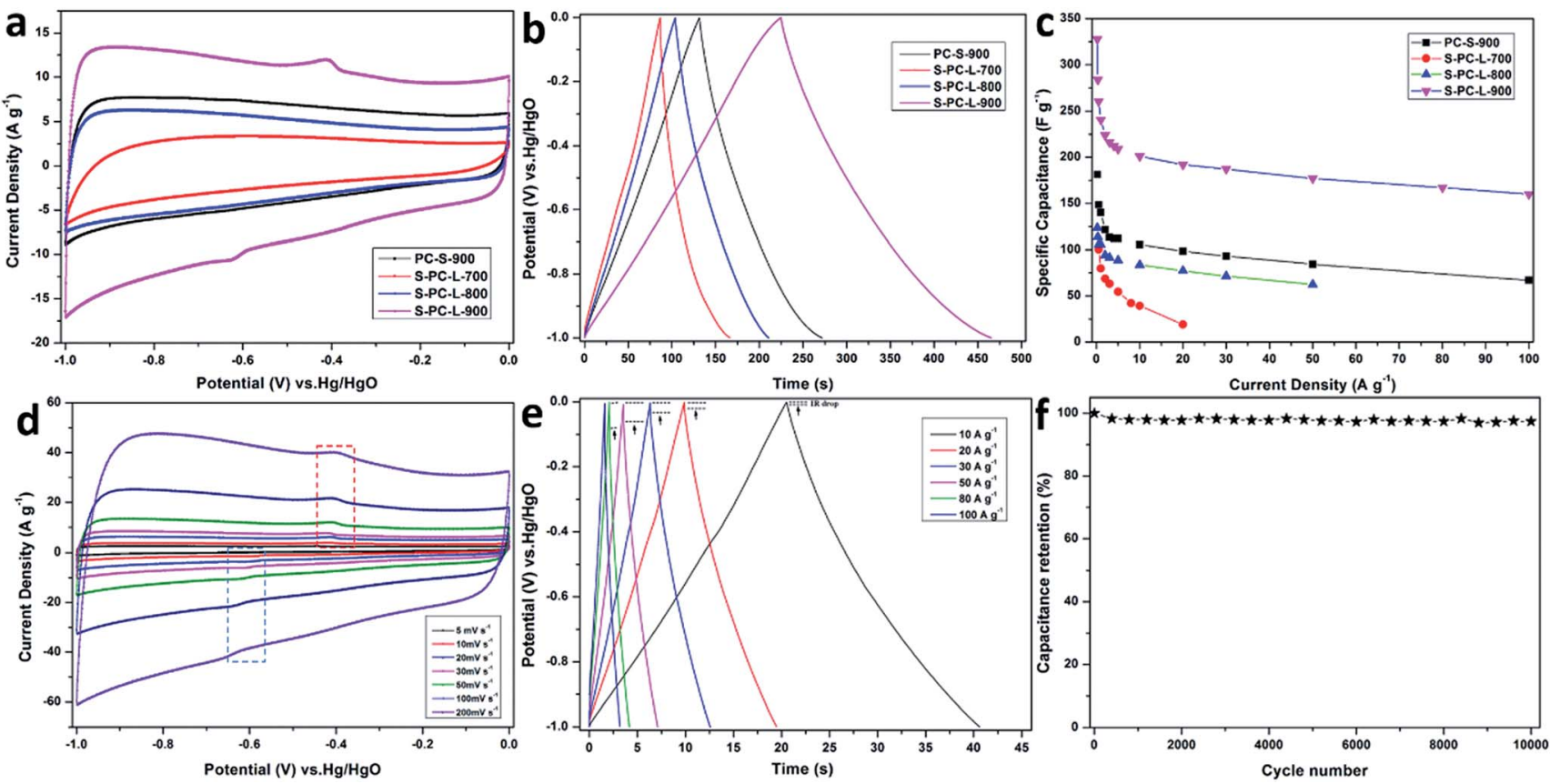

Fig. 5 Electrochemical performance of the samples in $6 \mathrm{M} \mathrm{KOH}$ aqueous electrolyte in a three-electrode system. (a) $\mathrm{CV}$ curves at $50 \mathrm{mV} \mathrm{s}^{-1}$, (b) galvanostatic charge-discharge curves at $1 \mathrm{~A} \mathrm{~g}^{-1}$, (c) specific capacitance at various current densities, (d) CV curves of S-PC-L-900 at various scan rates, (e) galvanostatic charge-discharge curves of S-PC-L-900 at various current densities, and (f) capacitance retention of S-PC-L-900 versus number of cycles at $200 \mathrm{mV} \mathrm{s}^{-1}$.

electron density could be located at the surface because of the electron-rich sulfur. The enhanced electric dipole moment enlarged the total polarization, resulting the larger electrolyte dielectric constant. Secondly, a series of redox faradic reactions are carried out on the S-doped carbon at the additional sulfone and sulfoxide species. The pseudo-capacitive properties are related with oxidized sulfur species but facilitated by thiophene sulfur. ${ }^{53}$

$\mathrm{CV}$ curves of all samples at scan rates from 5 to $200 \mathrm{mV} \mathrm{s}^{-1}$ are shown in Fig. 5d and S6a-c. $\dagger$ The results indicate that the CV curves of S-PC-L-700 are distorted from the rectangular shape. On the contrary, the CV curves of S-PC-L-800, -900, and PC-S-900 maintain a rectangular shape at various scan rates.
The rectangular shape at $200 \mathrm{mV} \mathrm{s}^{-1}$ and magnified CV curve area of S-PC-L-900 suggest high capacity for charge storage and remarkable rate performance. As shown in the GCD curves of the samples at a current density of $1 \mathrm{~A} \mathrm{~g}^{-1}$ (Fig. 5b), S-PC-L-900 shows the longest charge-discharge time.

GCD curves of samples of S-PC-L-900, -800, and PC-S-900 at high current density (10 to $100 \mathrm{~A} \mathrm{~g}^{-1}$; Fig. 5e, S5e and $\mathrm{f} \dagger$ ) are highly linear and symmetrical, indicating that they have excellent electrochemical reversibility and columbic efficiency. Compared with that of the other samples, the $I R$ drop of S-PC-L900 is inconspicuous even at high current. This may due to the low internal series resistance, which can be demonstrated by EIS (Fig. S6 $\dagger$ ). GCD curves for S-PC-L-700 (Fig. S5d $\dagger$ ) are 
extremely unsymmetrical, indicating inferior electrochemical property. Fig. $5 \mathrm{c}$ plots the gravimetric specific capacitance of all samples versus current density. S-PC-L-900 has the largest specific capacitance. The specific capacitances of S-PC-L-900 are $328,260,241$, and $210 \mathrm{~F} \mathrm{~g}^{-1}$ at current densities of $0.2,0.5,1$, and $5 \mathrm{~A} \mathrm{~g}^{-1}$, respectively. Otherwise, a specific capacitance of $192 \mathrm{~F} \mathrm{~g}^{-1}$ is obtainable even at $20 \mathrm{~A} \mathrm{~g}^{-1}$, showing excellent rate performance $(73 \%$ capacitance retention after current density expending 40 times). The specific capacitance of S-PC-L-900 is almost twice that of PC-S-900 $\left(98.4 \mathrm{~F} \mathrm{~g}^{-1}\right.$ at $20 \mathrm{~A} \mathrm{~g}^{-1} ; 66 \%$ capacitance retention) and more than double that of S-PC-L-800 (77.2 $\mathrm{F} \mathrm{g}^{-1}$ at $20 \mathrm{~A} \mathrm{~g}^{-1} ; 73 \%$ capacitance retention). These results indicate that introduction of sulfur ${ }^{7}$ and increasing the number of macropores increase the capacitive performance. The hierarchically S-doped macroporous frameworks can provide short and unimpeded diffusion channels for electrolyte ions even at high current density. Moreover, the cycling stability can be improved by the hierarchically S-doped macroporous frameworks. As shown in Fig. 5f, the capacitance retention for SPC-L-900 remains $97 \%$ after 10000 cycles at a scan rate of $200 \mathrm{mV} \mathrm{s}^{-1}$, indicating excellent cycling stability. Besides, we also investigate the capacitive performance of S-PC-L-900 in $\mathrm{H}_{2} \mathrm{SO}_{4}$ and $\mathrm{Na}_{2} \mathrm{SO}_{4}$ aqueous electrolyte. The CV curves of S-PC-L900 show a rectangular shape at a scan rate of $50 \mathrm{mV} \mathrm{s}^{-1}$ (Fig. S7a $\dagger$ ) and GCD curves are linear and symmetrical at current densities of $1 \mathrm{~A} \mathrm{~g}^{-1}$ (Fig. S7b $\dagger$ ). The results indicate that the S-PC-L-900 has good capacity for charge storage in both $\mathrm{H}_{2} \mathrm{SO}_{4}$ and $\mathrm{Na}_{2} \mathrm{SO}_{4}$ aqueous electrolyte. However, the S-PC-L-900 shows higher capacity in $\mathrm{H}_{2} \mathrm{SO}_{4}$ aqueous electrolyte than $\mathrm{Na}_{2} \mathrm{SO}_{4}$ aqueous electrolyte while both lower compared with $\mathrm{KOH}$ aqueous electrolyte (Fig. S7c $\dagger$ ).

In order to investigate the capacitive performance of the electrode in a complete cell, a symmetric supercapacitor was assembled by employing two S-PC-L-900 specimens in $6 \mathrm{M} \mathrm{KOH}$ aqueous electrolyte. Fig. 6a shows the CV curves of S-PC-L-900 at scan rates of 10 to $1000 \mathrm{mV} \mathrm{s}^{-1}$. Even at very high scan rate $\left(1000 \mathrm{mV} \mathrm{s}^{-1}\right)$, the $\mathrm{CV}$ curve is still rectangular, indicating ideal capacitive behavior and rate performance. Furthermore, the GCD curves (Fig. 6b) of the S-PC-L-900 supercapacitor even at extremely high current density (inset, 5-30 $\mathrm{A} \mathrm{g}^{-1}$ ) exhibit ideal symmetrical triangle without obvious IR drop, indicating excellent electrochemical reversibility and low internal resistance. ${ }^{10}$ The corresponding specific capacitance of S-PC-L-900 based on CV and GCD curves is shown in Fig. S8. $\dagger$ The maximum capacitance is $40.0 \mathrm{~F} \mathrm{~g}^{-1}$ at $10 \mathrm{mV} \mathrm{s}^{-1}$ and $49.8 \mathrm{~F} \mathrm{~g}^{-1}$ at $0.2 \mathrm{~A} \mathrm{~g}^{-1}$, respectively, higher than those for carbon-based symmetric supercapacitors. ${ }^{7}$

To obtain further insight into the electrochemical stability of the as-prepared S-PC-L-900 hybrid, the capacitor in $6 \mathrm{M} \mathrm{KOH}$ electrolyte was measured at $2 \mathrm{~A} \mathrm{~g}^{-1}$ over 10000 cycles. As shown
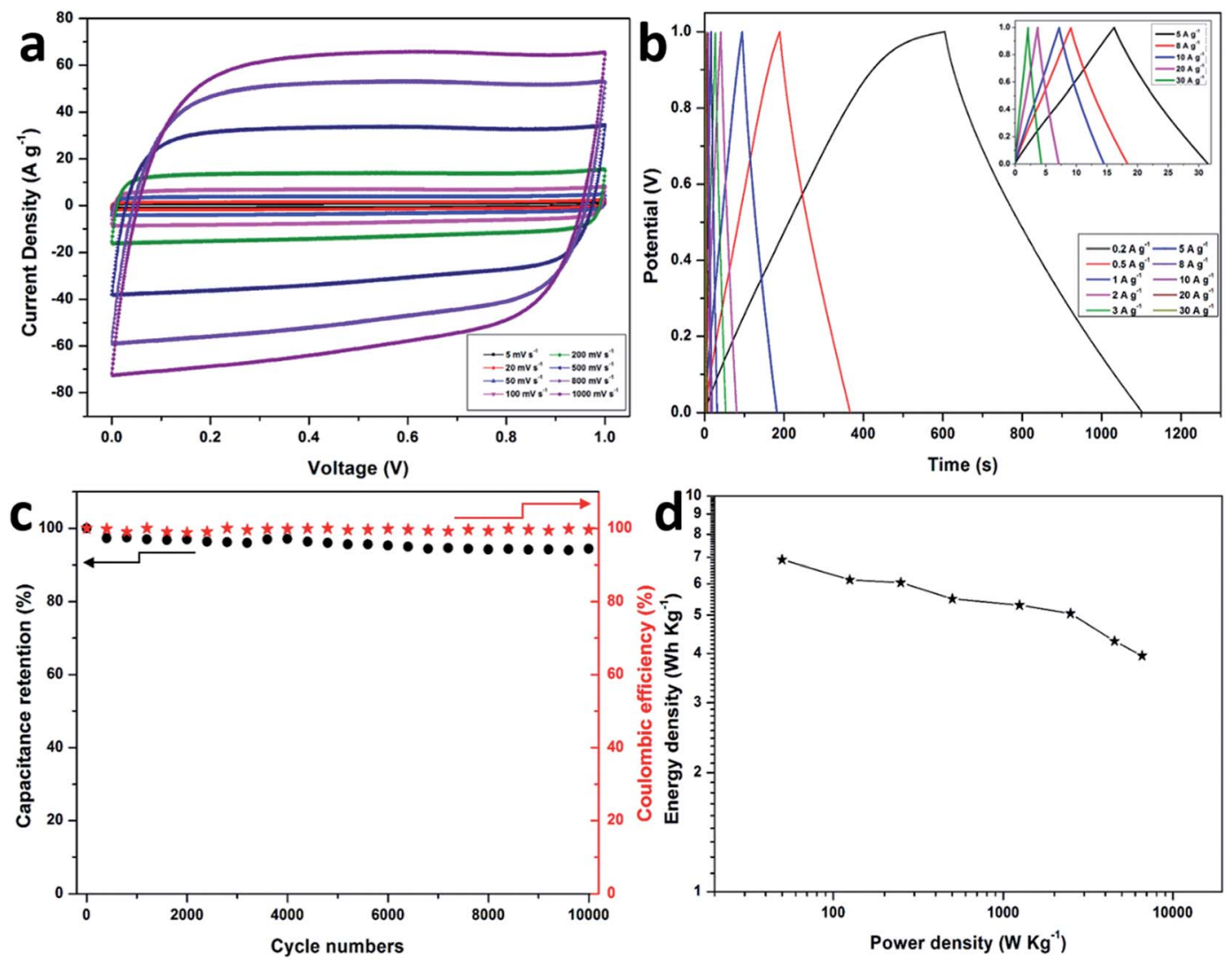

Fig. 6 Electrochemical performance of the S-PC-L-900 in $6 \mathrm{M} \mathrm{KOH}$ aqueous electrolyte in a two-electrode system. (a) CV curves at scan rates of 5 to $1000 \mathrm{mV} \mathrm{s}^{-1}$. (b) GCD curves at current densities of 0.2 to $30 \mathrm{~A} \mathrm{~g}^{-1}$; the inset shows GCD curves at current densities of 5 to $30 \mathrm{~A} \mathrm{~g}$. (c) Capacitance retention and coulombic efficiency versus the number of cycles at $2 \mathrm{~A} \mathrm{~g} \mathrm{~g}^{-1}$. (d) Ragone plots of the S-PC-L-900 symmetric cell. 
in Fig. 6c, the capacitance deterioration of S-PC-L-900 capacitor is less than $6 \%$ after 10000 cycles, and the coulombic efficiency remains at nearly $100 \%$. Moreover, the S-PC-L-900 capacitor shows a high energy density $\left(6.9 \mathrm{~W} \mathrm{~h} \mathrm{~kg}^{-1}\right)$ at a power density of $50 \mathrm{~W} \mathrm{~kg}^{-1}$ (Fig. 6d), higher than that of previously reported carbon-based supercapacitors. ${ }^{\mathbf{1 0 , 5 4}}$ The power densities of the carbon-based supercapacitors are shown in Fig. 6d. This improved performance can be explained by the S-doped macroporous structure, which could serve as ion-buffering reservoirs and affect ion pathways. ${ }^{55}$

\section{Conclusions}

In summary, we have fabricated hierarchically S-doped porous carbon through a simple template technique using by-products lignin as carbon and sulfur precursor. The obtained carbon sheet consists of interconnected open macropores, mesopores, and micropores. Because of its high surface area and wide pore size distribution, the as-obtained S-PC-L-900 exhibits a high specific capacitance $\left(328 \mathrm{~F} \mathrm{~g}^{-1}\right)$ at $0.2 \mathrm{~A} \mathrm{~g}^{-1}$, as well as excellent rate performance $(73 \%$ capacitance retention after current density increase from 0.5 to $\left.20 \mathrm{~A} \mathrm{~g}^{-1}\right)$ and cycling stability $(97 \%$ capacitance retention after 10000 cycles). Furthermore, the symmetric supercapacitor based on S-PC-L-900 delivers a high energy density $\left(6.9 \mathrm{~W} \mathrm{~h} \mathrm{~kg}^{-1}\right)$ at $50 \mathrm{~W} \mathrm{~kg}^{-1}$. This high electrocapacitive performance indicates that our hierarchically $\mathrm{S}$ doped porous carbon provide a low-cost and ecofriendly biomaterial for next-generation energy storage applications.

\section{Acknowledgements}

This work is financially supported by the link project of the National Natural Science Foundation of China and Guangdong Province (No. U1401246), the Science and Technology Program of Guangdong Province of China (Grant No. 2014B010106005, 2015B010135011, 2015A050502047, 2016A020221031), the Science and Technology Program of Guangzhou City of China (Grant No. 201508030018, 2016201604030040), the National Nature Science Foundation of China Key Projects of Guangdong Joint Fund (U1401246) and the National Nature Science Foundation of China (51276044, 51302043).

\section{References}

1 Y. Sun, Q. Wu and G. Shi, Energy Environ. Sci., 2011, 4, 1113. 2 Y. Meng, Y. Zhao, C. Hu, H. Cheng, Y. Hu, Z. Zhang, G. Shi and L. Qu, Adv. Mater., 2013, 25, 2326.

3 C. Long, X. Chen, L. Jiang, L. Zhi and Z. Fan, Nano Energy, 2015, 12, 141.

4 F. Zhang, T. Zhang, X. Yang, L. Zhang, K. Leng, Y. Huang and Y. Chen, Energy Environ. Sci., 2013, 6, 1623.

5 H. Jiang, S. Lee and C. Li, Energy Environ. Sci., 2013, 6, 41. 6 S. Faraji and F. N. Ani, J. Power Sources, 2014, 263, 338.

7 Y. Li, G. Wang, T. Wei, Z. Fan and P. Yan, Nano Energy, 2016, 19, 165.

8 Y. S. Yun, M. H. Park, S. J. Hong, M. E. Lee, Y. W. Park and H. J. Jin, ACS Appl. Mater. Interfaces, 2015, 7, 3684.
9 J. Yin, D. Zhang, J. Zhao, X. Wang, H. Zhu and C. Wang, Electrochim. Acta, 2014, 136, 504.

10 Q. Xie, A. Zheng, S. Zhai, S. Wu, C. Xie, Y. Zhang and Y. Guan, J. Solid State Electrochem., 2016, 20, 449.

11 C. Portet, G. Yushin and Y. Gogotsi, J. Electrochem. Soc., 2008, 155, A531.

12 M. Lee, G. Kim, H. Don Song, S. Park and J. Yi, Nanotechnology, 2014, 25, 345601.

13 B. Xu, S. Hou, G. Cao, F. Wu and Y. Yang, J. Mater. Chem., 2012, 22, 19088.

14 O. Ioannidou and A. Zabaniotou, Renewable Sustainable Energy Rev., 2007, 11, 1966.

15 D. Jiménez-Cordero, F. Heras, M. A. Gilarranz and E. Raymundo-Piñero, Carbon, 2014, 71, 127.

16 X. He, P. Ling, M. Yu, X. Wang, X. Zhang and M. Zheng, Electrochim. Acta, 2013, 105, 635.

17 Y. Liu, Z. Shi, Y. Gao, W. An, Z. Cao and J. Liu, ACS Appl. Mater. Interfaces, 2016, 8, 28283.

18 H. Pińkowska, P. Wolak and A. Złocińska, Chem. Eng. J., 2012, 187, 410.

19 R. J. A. Gosselink, W. Teunissen, J. E. G. van Dam, E. de Jong, G. Gellerstedt, E. L. Scott and J. P. M. Sanders, Bioresour. Technol., 2012, 106, 173.

20 S. Some, J. Kim, K. Lee, A. Kulkarni, Y. Yoon, S. Lee, T. Kim and H. Lee, Adv. Mater., 2012, 24, 5481.

21 H. L. Poh, P. Šimek, Z. Sofer and M. Pumera, ACS Nano, 2013, 7, 5262.

22 H. Liu, Y. Liu and D. Zhu, J. Mater. Chem., 2011, 21, 3335.

23 X. Yu and H. S. Park, Carbon, 2014, 77, 59.

24 C. Long, D. Qi, T. Wei, J. Yan, L. Jiang and Z. Fan, Adv. Funct. Mater., 2014, 24, 3953.

25 J. S. Han, H. S. Kim and J. Neggers, Advances in Difference Equations, 2012, 2012, 19.

26 L. F. Chen, Z. H. Huang, H. W. Liang, H. L. Gao and S. H. Yu, Adv. Funct. Mater., 2014, 24, 5104.

27 Z. Yang, H. Nie, X. Chen, X. Chen and S. Huang, J. Power Sources, 2013, 236, 238.

28 Y. Yan, Y. Yin, S. Xin, Y. Guo and L. Wan, Chem. Commun., 2012, 48, 10663.

29 L. Yue, H. Zhong, D. Tang and L. Zhang, J. Solid State Electrochem., 2013, 17, 961.

30 G. Hasegawa, M. Aoki, K. Kanamori, K. Nakanishi, T. Hanada and K. Tadanaga, J. Mater. Chem., 2011, 21, 2060.

31 W. Si, J. Zhou, S. Zhang, S. Li, W. Xing and S. Zhuo, Electrochim. Acta, 2013, 107, 397.

32 M. Sevilla, A. B. Fuertes and R. Mokaya, Int. J. Hydrogen Energy, 2011, 36, 15658.

33 Z. Yang, Z. Yao, G. Li, G. Fang, H. Nie, Z. Liu, X. Zhou, X. Chen and S. Huang, ACS Nano, 2012, 6, 205.

34 F. Shahzad, P. Kumar, S. Yu, S. Lee, Y. Kim, S. Man and C. Min, J. Mater. Chem. C, 2015, 3, 9802.

35 M. Sevilla and A. B. Fuertes, ACS Nano, 2014, 8, 5069.

36 T. Liu, E. Liu, R. Ding, Z. Luo, T. Hu and Z. Li, Electrochim. Acta, 2015, 173, 50.

37 D. Kawashima, T. Aihara, Y. Kobayashi, T. Kyotani and A. Tomita, Chem. Mater., 2000, 12, 3397. 
38 W. Gao, X. Feng, T. Zhang, H. Huang, J. Li and W. Song, ACS Appl. Mater. Interfaces, 2014, 6, 19109.

39 F. Shahzad, P. Kumar, Y. H. Kim, S. M. Hong and C. M. Koo, ACS Appl. Mater. Interfaces, 2016, 8, 9361.

40 K. Krishnamoorthy, M. Veerapandian, K. Yun and S.-J. Kim, Carbon, 2013, 53, 38.

41 Y. Zhan, J. Huang, Z. Lin, X. Yu, D. Zeng, X. Zhang, F. Xie, W. Zhang, J. Chen and H. Meng, Carbon, 2015, 95, 930.

42 W. Chen, J. Shi, T. Zhu, Q. Wang, J. Qiao and J. Zhang, Electrochim. Acta, 2015, 177, 327.

43 W. Chaikittisilp, M. Hu, H. Wang, H. Huang, T. Fujita, K. C.-W. Wu, L. Chen, Y. Yamauchi and K. Ariga, Chem. Commun., 2012, 48, 7259.

44 A. Sadezky, H. Muckenhuber, H. Grothe, R. Niessner and U. Pöschl, Carbon, 2005, 43, 1731.

45 Z. Yang, J. Guo, S. K. Das, Y. Yu, Z. Zhou, H. D. Abruna and L. A. Archer, J. Mater. Chem. A, 2013, 1, 1433.

46 Z. Li, Z. Xu, X. Tan, H. Wang, C. M. B. Holt, T. Stephenson, B. C. Olsen and D. Mitlin, Energy Environ. Sci., 2013, 6, 871878.
47 W. Ai, Z. Luo, J. Jiang, J. Zhu, Z. Du, Z. Fan, L. Xie, H. Zhang, W. Huang and T. Yu, Adv. Mater., 2014, 26, 6186.

48 M. Seredych, E. Rodríguez-Castellón, M. J. Biggs, W. Skinner and T. J. Bandosz, Carbon, 2014, 78, 540.

49 S.-A. A. Wohlgemuth, R. J. White, M.-G. G. Willinger, M.-M. M. Titirici and M. Antonietti, Green Chem., 2012, 14, 1515.

50 S. Zhang, F. Yao, L. Yang, F. Zhang and S. Xu, Carbon, 2015, 93, 143.

51 S. Gao, H. Liu, K. Geng and X. Wei, Nano Energy, 2015, 12, 785.

52 R. B. Rakhi, W. Chen, D. Cha and H. N. Alshareef, J. Mater. Chem., 2011, 21, 16197.

53 X. Zhao, Q. Zhang, C. M. Chen, B. Zhang, S. Reiche, A. Wang, T. Zhang, R. Schlögl and D. Sheng Su, Nano Energy, 2012, 1, 624.

54 T. Y. Kim, H. W. Lee, M. Stoller, D. R. Dreyer, C. W. Bielawski, R. S. Ruoff and K. S. Suh, ACS Nano, 2011, 5, 436.

55 B. G. Choi, M. Yang, W. H. Hong, J. W. Choi and Y. S. Huh, ACS Nano, 2012, 6, 4020. 